\title{
Novel strategies for preparation and characterization of functional polymer-metal nanocomposites for electrochemical applications*
}

\author{
Dmitri N. Muraviev ${ }^{1, \ddagger}$, Patricia Ruiz ${ }^{1}$, Maria Muñoz ${ }^{1}$, \\ and Jorge Macanás ${ }^{2}$ \\ ${ }^{1}$ Department of Chemistry, Autonomous University of Barcelona, 08193 Bellaterra, \\ Barcelona, Spain; ${ }^{2}$ Université de Toulouse, INP, UPS, CNRS, Laboratory of \\ Chemical Engineering, F31062 Toulouse Cedex 09, France
}

\begin{abstract}
Stabilization of metal nanoparticles (MNPs) in polymeric matrices of different types has proven to be one of the most promising strategies to prevent their aggregation and to retain their properties. Polymer-stabilized MNPs (PSMNPs) and those based on polymermetal nanocomposite materials are starting to find wide application in various fields of science and technology. In this paper, we demonstrate that metal-polymer nanocomposite membranes (MPNCMs) containing MNPs can easily be prepared in an ion-exchange such as, for example, sulfonated polyetherether ketone (SPEEK) matrix by using the polymeric membranes as nanoreactors for synthesis and to characterize the composition and structure of the formed MNPs. Metal ions (or metal ion complexes) are first incorporated into the polymeric matrix where they undergo reduction, leading to formation of corresponding MPNCMs. Since this technique allows successive metal loading-reduction cycles to be carried out, it enables synthesis of both monometallic and bimetallic (e.g., core-shell) MNPs. The proposed approach is illustrated by synthesis and characterization of MPNCMs containing both monometallic and bimetallic core-shell MNPs, formed by combinations of Pd, $\mathrm{Pt}, \mathrm{Co}, \mathrm{Ni}$, and $\mathrm{Cu}$, along with their application in electrochemical sensor and biosensor constructions.
\end{abstract}

Keywords: sensors; biosensors; core-shell nanoparticles; platinum; palladium.

\section{INTRODUCTION}

The synthesis and characterization of metal nanoparticles (MNPs) has attracted great interest of scientists and technologists during recent years, owing to their unique physical and chemical properties, which differ substantially from those of both bulk material and single atoms. These properties facilitate various practical applications of MNPs including catalysis- and electrocatalysis-based processes, which occur, for example, in fuel cells of different types or in various sensing devices (e.g., amperometric sensors and biosensors). A major drawback, which still limits their wider application, is insufficient stability of MNPs, exemplified by their pronounced tendency to aggregate, with resultant loss of nano-

\footnotetext{
*Paper based on a presentation at the $3^{\text {rd }}$ International Symposium on Novel Materials and Their Synthesis (NMS-III) and the $17^{\text {th }}$ International Symposium on Fine Chemistry and Functional Polymers (FCFP-XVII), 17-21 October 2007, Shanghai, China. Other presentations are published in this issue, pp. 2231-2563.

¥Corresponding author
} 
metric size and special properties in the coalescent state. Thus, stabilization is necessary during growth in order to prevent aggregation, to allow their dissolution/dispersion in some solvents and to protect the MNPs from Oswald ripening [1]. Oswald ripening is the growth mechanism whereby small particles dissolve, and are consumed by larger nanoparticles. Stabilization of MNPs in polymeric matrices of different types has proven to be one of the most promising strategies to prevent their aggregation and to conserve their properties [2-10]. Polymer-stabilized MNPs (PSMNPs) and those based on polymermetal nanocomposite materials are starting to find wide applications in various fields of science and technology for the following reasons: (i) the presence of MNPs in a polymeric matrix enhances the mass transfer parameters of the nanocomposite and therefore, for example, the response time of chemical sensors [11] and electrochemical biosensors [12], due to the shortening of electron-hopping or -tunneling distances; (ii) the PSMNP-stabilizing polymer substantially simplifies further processing of nanoparticles, through solubilization in certain solvents, thereby making it possible to work with PSMNP solutions (PSMNP inks); and (iii) PSMNP inks can easily be deposited on various supports, and subsequent evaporation of the solvent results in formation of polymer-metal nanocomposite membranes (e.g., on electrode surfaces, catalyst supports, etc.). In the case of biosensors, the polymer matrix makes it possible to immobilize enzymes, and PSMNPs can also act as mediators due to their ability to interact with one of the products of enzymatic reaction (e.g., $\mathrm{H}_{2} \mathrm{O}_{2}$ ).

The electrochemical applications of MNPs (e.g., in sensor and biosensor constructions) are, in many instances, based on the use of noble metals for their well-known electrocatalytic properties. The main drawback associated with the use of these metals is their high cost. For this reason, one of the most important challenges is to decrease the loading of noble metal in the synthesis of MNPs without impairing the performance of nanocatalysts. This problem can be solved through the use of core-shell nanoparticles, comprising a cheap metal core coated with a thin noble metal shell. Core-shell MNPs can be formed using different metals depending on their further application. The coating (shell formation) influences the charge, functionality, or stability of core-MNPs [13]. This enables properties of the final material to be 'tuned' in accordance with its practical application. For example, Pt coating of Co-core MNPs produces nanoparticles with catalytic and magnetic properties, which can be easily recovered and reused or reprocessed.

The main methods for preparation of PSMNPs include chemical and physical techniques [14]. Chemical methods are usually the less expensive option of the two, since they generally do not require complex and costly equipment. One example of a chemical technique is the intermatrix synthesis (IMS) of PSMNPs [2,7,8,15-17]. The IMS technique is based on the use of functional polymers (FPs), the matrices of which contain ionic functional groups, and can be used as a nanoreactor to synthesize MNPs with desired properties and of required structure.

In this paper, we demonstrate that metal-polymer nanocomposite membranes (MPNCMs) containing MNPs can easily be prepared using a sulfonated polyetherether ketone (SPEEK) polymeric matrix. SPEEK membranes facilitate synthesis and enable the composition and architecture of the formed MNPs to be characterized. Metal ions (or metal ion complexes) are first incorporated in the polymeric matrix, where they undergo reduction leading to formation of the corresponding MPNCMs. Since this technique allows successive metal loading-reduction cycles to be carried out, it enables synthesis of both monometallic and bimetallic (e.g., core-shell) MNPs $[16,18,19]$. The proposed approach is illustrated by synthesis and characterization of MPNCMs containing both mono- and bimetallic core-shell MNPs, formed by combinations of $\mathrm{Pd}, \mathrm{Pt}, \mathrm{Co}, \mathrm{Ni}$, and $\mathrm{Cu}$.

\section{EXPERIMENTAL}

Metal salts $\mathrm{KCl}, \mathrm{NaBH}_{4}, \mathrm{CuSO}_{4} \cdot 5 \mathrm{H}_{2} \mathrm{O},\left[\mathrm{Pd}\left(\mathrm{NH}_{3}\right)_{4}\right] \mathrm{Cl}_{2}$, $\left[\mathrm{Pt}\left(\mathrm{NH}_{3}\right)_{4}\right]\left(\mathrm{NO}_{3}\right)_{2}, \mathrm{Co}\left(\mathrm{NO}_{3}\right)_{2} \cdot 6 \mathrm{H}_{2} \mathrm{O}$, and $\mathrm{Ni}\left(\mathrm{NO}_{3}\right)_{2} \cdot 6 \mathrm{H}_{2} \mathrm{O}$ (all from Aldrich, Germany), acids, and organic solvents (all from Panreac, S.A. Spain) of p.a. grade were used as received. Polymers (polyetherether ketone, PEEK, Goodfellow, and 
polyethylenimne, PEI, Aldrich) were also used without any pretreatment. Glucose oxidase (GOx) was purchased from Fluka. Bidistilled water was used in all experiments carried out.

Graphite-epoxy composite electrodes (GECEs) [20] were prepared by using graphite powder with a particle size of $50 \mu \mathrm{m}$ (BDH, UK), Epotek H77, epoxy resin and the hardener (both from Epoxy Technology, USA). Graphite powder was hand-mixed with epoxy resin and hardener in a ratio of 1:4 (w/w). The resulting paste was placed into a poly(vinyl chloride) (PVC) cylindrical sleeve body (6 $\mathrm{mm}$ i.d.), which had an inner electrical $\mathrm{Cu}$ contact, to a depth of $3 \mathrm{~mm}$. The conducting composite material glued to the $\mathrm{Cu}$ contact was cured at $40{ }^{\circ} \mathrm{C}$ during 7 days. Before use, the surface of electrode was washed with doubly distilled water and then thoroughly polished first with abrasive paper and finally with alumina paper (polishing strips 301044-001, Moyco Precision Abrasives, Inc.).

\section{Methods}

Polyetheretherketone was sulfonated using sulfuric acid by following the procedure similar to that described elsewhere [21]. The dried polymer $(20 \mathrm{~g})$ was dissolved in concentrated sulfuric acid $(1 \mathrm{~L})$ under vigorous stirring for $100 \mathrm{~h}$. After that, the mixture was poured into $10 \mathrm{~L}$ of iced water where the polymer precipitated. Polymer-rich part was then washed repeatedly with distilled water until the neutral $\mathrm{pH}$. Then, the polymer was filtered, dried, and dissolved in dimethylformamide (DMF) before casting a membrane by solvent evaporation.

The synthesis of PSMNPs in SPEEK membranes was carried out by IMS technique, which consists of two sequential steps: (1) the loading of sulfonic groups with metal ions $\left(\left[\mathrm{Pd}\left(\mathrm{NH}_{3}\right)_{4}\right]^{2+}\right.$, $\left[\mathrm{Pt}\left(\mathrm{NH}_{3}\right)_{4}\right]^{2+}, \mathrm{Cu}^{2+}, \mathrm{Co}^{2+}, \mathrm{Ni}^{2+}$, or a mixture of $\mathrm{Co}^{2+}$ and $\mathrm{Ni}^{2+}$ with ratio $1: 1$, using a $0.01 \mathrm{M}$ solution for $\left[\mathrm{Pd}\left(\mathrm{NH}_{3}\right)_{4}\right] \mathrm{Cl}_{2}$ and $\left[\mathrm{Pt}\left(\mathrm{NH}_{3}\right)_{4}\right]\left(\mathrm{NO}_{3}\right)_{2}$ and $0.1 \mathrm{M}$ solution for $\mathrm{CuSO} \mathrm{Cu}_{4} \cdot 5 \mathrm{H}_{2} \mathrm{O}, \mathrm{Co}\left(\mathrm{NO}_{3}\right)_{2} \cdot 6 \mathrm{H}_{2} \mathrm{O}$, and $\mathrm{Ni}\left(\mathrm{NO}_{3}\right)_{2} \cdot 6 \mathrm{H}_{2} \mathrm{O}$ ) followed by (2) the reduction of metals inside the matrix by using $0.1 \mathrm{M}$ aqueous $\mathrm{NaBH}_{4}$ solution (IMS stage). After rinsing with bidistilled water, the PSMNP-containing membranes were air-dried at room temperature. The core-shell MNPs were synthesized by using two sequential metal loading-reduction cycles. The core-MNPs were prepared within the first cycle while the second cycle served for the coating of preformed MNPs with another metal. The PSMNP inks prepared by dissolution of SPEEK-MNP-nanocomposite membranes in DMF (5\% w/w) were drop-by-drop deposited onto the surface of GECE followed by air-drying at room temperature to form a membrane. Samples of PSMNP inks after appropriate dilution were used for transmission electron microscopy (TEM) analysis.

The biosensors were prepared by using the layer-by-layer deposition technique partially similar to that described elsewhere [22]. The bio-recognition layer was obtained by alternate dip-coatings of GECEs modified with SPEEK-Pt@Cu-PSMNP membrane in $6.0 \mathrm{mg} / \mathrm{mL}$ PEI and $8.0 \mathrm{mg} / \mathrm{mL}$ GOx solutions followed by washing with water after each step. The layer deposition time in all cases was $1 \mathrm{~h}$. PEI and GOx solutions were prepared in a $0.050 \mathrm{M}$ phosphate buffer solution at $\mathrm{pH}=7.0$.

The size and structure of PSMNPs was characterized by transmission electron microscope (JEOL 2011, Jeol, Ltd.). The current intensities in amperometric detection of $\mathrm{H}_{2} \mathrm{O}_{2}$ and glucose were measured by using a PC-controlled Model 800B Electrochemical Analyzer ( $\mathrm{CH}$ Instruments, USA) supplied with an auxiliary Pt electrode 52-671 (Crison) and a reference $\mathrm{Ag} / \mathrm{AgCl}$ electrode (Orion 900200).

\section{RESULTS AND DISCUSSION}

The IMS technique makes it possible to produce both MNPs and the corresponding polymer-metal nanocomposites by using a simple procedure. A schematic diagram of this technique is shown in Fig. 1. The PSMNP formation procedure includes two stages: (1) loading of sulfonic groups of SPEEK membrane with metals cations (e.g., $\mathrm{Cu}^{2+}, \mathrm{Co}^{2+}, \mathrm{Ni}^{2+}$, etc.) followed by (2) metal reduction inside the SPEEK matrix which results in the formation of monometallic MNPs. The repetitive (sequential) metal loading-reduction cycle when using another metal results in the coating of preformed MNPs with the 


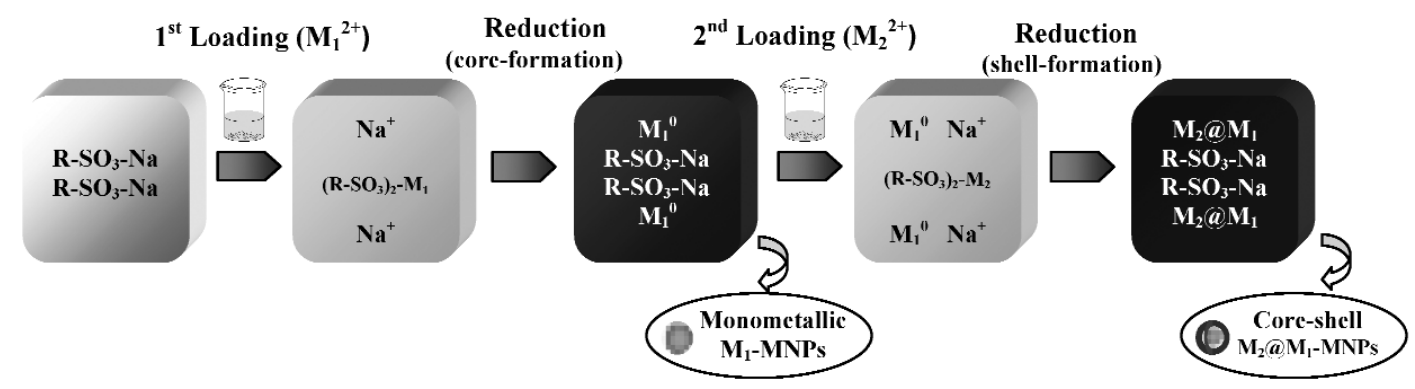

Fig. 1 Schematic diagram of IMS of mono- or bimetallic (core-shell) PSMNPs inside polymeric matrix of SPEEK, which consists of sequential loading and reduction cycles.

second metal shell. In other words, two sequential metal loading-reduction cycles by using two different metals allows for the formation of core-shell MNPs.

The IMS of PSMNPs inside the stabilizing polymeric matrix (SPEEK in our case) can be described by the following reactions:

1. Monometallic MNPs:

a) $\quad 1^{\text {st }}$ metal loading

$$
2 \mathrm{R}-\mathrm{SO}_{3}{ }^{-} \mathrm{Na}^{+}+\mathrm{M}_{1}{ }^{2+} \rightarrow\left(\mathrm{R}-\mathrm{SO}_{3}^{-}\right)_{2} \mathrm{M}_{1}{ }^{2+}+2 \mathrm{Na}^{+}
$$

b) $1^{\text {st }}$ metal reduction:

$$
\left(\mathrm{R}-\mathrm{SO}_{3}{ }^{-}\right)_{2} \mathrm{M}_{1}{ }^{2+}+2 \mathrm{NaBH}_{4}+6 \mathrm{H}_{2} \mathrm{O} \rightarrow 2 \mathrm{R}_{-} \mathrm{SO}_{3}{ }^{-} \mathrm{Na}^{+}+7 \mathrm{H}_{2}+2 \mathrm{~B}(\mathrm{OH})_{3}+\mathrm{M}_{1}{ }^{\mathrm{O}}
$$

2. Bimetallic core-shell MNPs:

a) $2^{\text {nd }}$ metal loading:

$$
2 \mathrm{R}-\mathrm{SO}_{3}{ }^{-} \mathrm{Na}^{+}+\mathrm{M}_{1}{ }^{0}+\mathrm{M}_{2}{ }^{2+} \rightarrow\left(\mathrm{R}^{-} \mathrm{SO}_{3}^{-}\right)_{2} \mathrm{M}_{2}{ }^{2+}+\mathrm{M}_{1}{ }^{0}+2 \mathrm{Na}^{+}
$$

b) $2^{\text {nd }}$ metal reduction:

$$
\begin{aligned}
& \left(\mathrm{R}-\mathrm{SO}_{3}{ }^{-}\right)_{2} \mathrm{M}_{2}{ }^{2+}+\mathrm{M}_{1}{ }^{0}+2 \mathrm{NaBH}_{4}+6 \mathrm{H}_{2} \mathrm{O} \rightarrow 2 \mathrm{R}_{-} \mathrm{SO}_{3}{ }^{-} \mathrm{Na}^{+}+7 \mathrm{H}_{2}+ \\
& 2 \mathrm{~B}(\mathrm{OH})_{3}+\mathrm{M}_{2} @ \mathrm{M}_{1}
\end{aligned}
$$

Metal reduction reactions are essentially the sum of the following processes:

$$
\begin{aligned}
& \left(\mathrm{R}_{-} \mathrm{SO}_{3}{ }^{-}\right)_{2} \mathrm{M}_{1}{ }^{2+}+2 \mathrm{Na}^{+} \rightarrow 2 \mathrm{R}-\mathrm{SO}_{3}{ }^{-} \mathrm{Na}^{+}+2 \mathrm{M}_{1}{ }^{2+} \\
& \mathrm{M}_{1}{ }^{2+}+\mathrm{BH}_{4}^{-}+6 \mathrm{H}_{2} \mathrm{O} \rightarrow \mathrm{M}_{1}{ }^{0}+7 \mathrm{H}_{2}+2 \mathrm{~B}(\mathrm{OH})_{3}
\end{aligned}
$$

As seen from the above reaction schemes (see, e.g., reaction 4) after each loading-reduction cycle, the functional groups of SPEEK appear to be completely regenerated (e.g., are converted back into Na-form). Therefore, the polymer appears to be essentially prepared (after complete removal of the reducing agent by washing with distilled water) for the next cycle. Note that the number of such cycles is not limited by two and can be, for example, extended to three or more, which gives a possibility to synthesize MNPs of more complex architecture (e.g., core-sandwich, onion-like, and so on).

The IMS technique (eqs. 1-4) was applied to synthesize both monometallic (Co-, Ni-, $\mathrm{Cu}-, \mathrm{Pd}-$, and Pt-MNPs) and bimetallic alloy (Co-Ni-MNPs), bimetallic core-shell (Co-, $\mathrm{Ni}$-, $\mathrm{Cu}$-core MNPs coated with Pd- or Pt-shell) and tri-metallic core-shell MNPs (bimetallic Co-Ni-core coated with Pt- or Pd-shell). The main advantage of the core-shell MNPs with platinum group metals (PGM) shell (e.g., $\mathrm{Pt} @ \mathrm{Cu}$ and Pd@Cu) is their far lower cost due to the lower PGM loading in comparison with monometallic PGM-MNPs. This advantage can also be achieved if at the same PGM loading the catalytic activity of core-shell MNPs is higher than that of their corresponding monometallic analogs. 
Moreover, the ferromagnetic nanocatalysts (MNPs with ferromagnetic core coated by a PGM shell, such asPt@Co,Pd@Co,Pt@Ni,Pd@Ni, and Pd@Co-Ni) have an additional advantage due to their magnetic properties. Indeed, their leaching into the environment can be easily prevented by using an electromagnetic "trap". This advantage is particularly important as the growing uncontrolled appearance of man-made nanoparticles of different types in the environment is considered to be a very serious modern ecological problem [23]. On the other hand, the PGM-containing MNPs recovered from the electromagnetic trap can be reused or reprocessed, which is also important both from the economical and the ecological viewpoints.

\section{Microscopic characterization}

Typical TEM images of PSMNPs under study are shown in Fig. 2. As seen, the shape of nanoparticles in all cases is close to the spherical one, except Pt-MNPs, which have an irregular shape and a far bigger size. For these reasons, one can expect an insufficiently high electrocatalytic activity of Pt-PSMNPs due to the low value of their surface area. The same partially concerns Pd-PSMNPs, the size of which appears to be higher than that of, for example, Pd@Cu-PSMNPs; although, unlike Pt-MNPs, Pd nanoparticles have nearly spherical shape. An average size of majority of bimetallic core-shell MNPs does not exceed $6 \mathrm{~nm}$ (see Table 1). In all cases, MNPs are well separated from each other and do not form any visible aggregates, which testifies that SPEEK matrix is characterized by a very good stabilizing efficiency toward nanoparticles synthesized by IMS technique. The bimetallic nature of coreshell nanoparticles is confirmed by the results of energy-dispersive spectrometry (EDS) analysis of PSMNP samples, some of which are shown in Fig. 3 along with the corresponding TEM images.

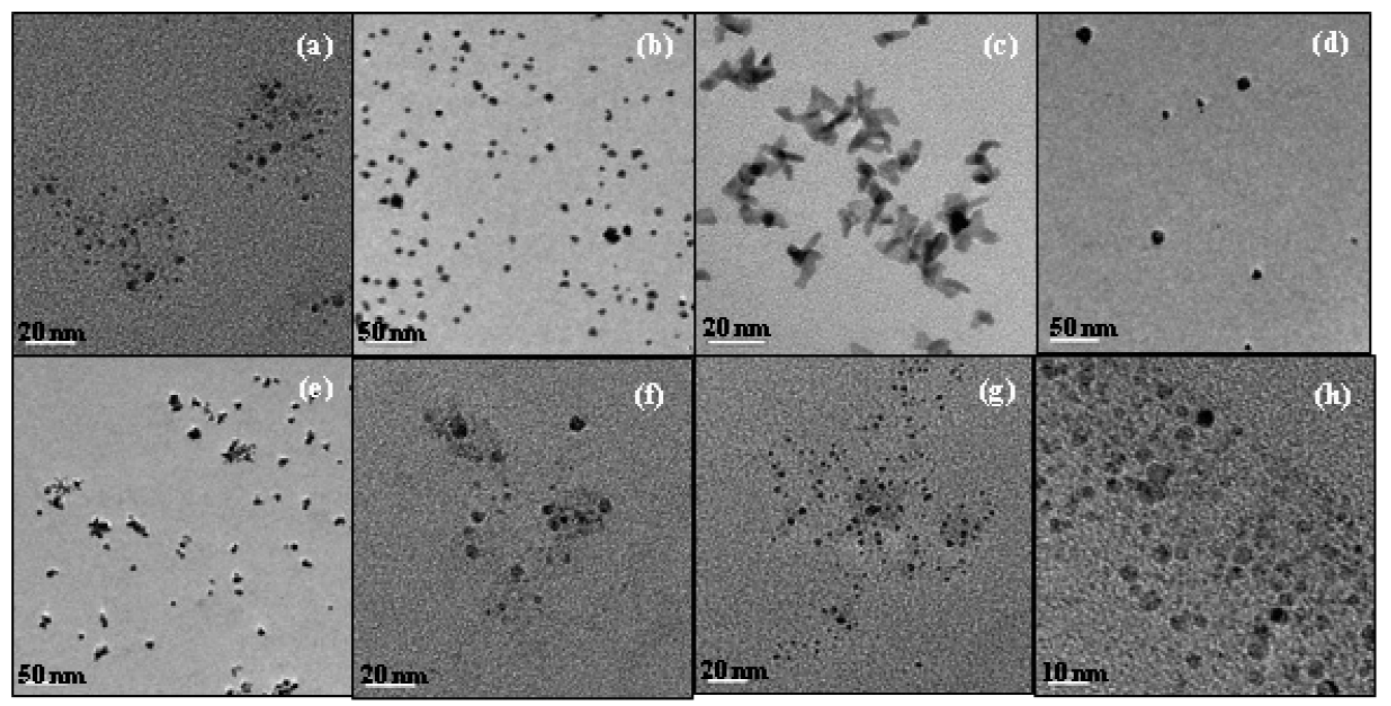

Fig. 2 Typical TEM images of Pt@Ni- (a), Pd- (b), Pt- (c), Pd@Co- (d), Pt@Co-Ni (e), Pd@Co-Ni (f), Ni- (g), and Cu-PSMNPs (h) (see text). 
Table 1 Parameters of histograms corresponding to different PSMNP samples.

\begin{tabular}{lcccc}
\hline $\begin{array}{l}\text { SPEEK-MNP } \\
\text { sample }\end{array}$ & $a$ & $d_{\mathrm{m}}(\mathrm{nm})$ & $\sigma$ & $r^{2}$ \\
\hline $\mathrm{Ni}$ & $93 \pm 2$ & $2.57 \pm 0.02$ & $0.59 \pm 0.02$ & 0.997 \\
$\mathrm{Pt} @ \mathrm{Co}$ & $62 \pm 4$ & $2.28 \pm 0.04$ & $0.62 \pm 0.04$ & 0.987 \\
$\mathrm{Pt} @ \mathrm{Ni}$ & $54 \pm 1$ & $2.16 \pm 0.01$ & $0.40 \pm 0.01$ & 0.997 \\
$\mathrm{Pt} @ \mathrm{Co}-\mathrm{Ni}$ & $26.4 \pm 0.8$ & $5.49 \pm 0.03$ & $0.99 \pm 0.03$ & 0.985 \\
$\mathrm{Pd} @ \mathrm{Co}$ & $38 \pm 3$ & $5.60 \pm 0.01$ & $1.6 \pm 0.1$ & 0.978 \\
$\mathrm{Pd} @ \mathrm{Ni}$ & $40 \pm 3$ & $1.22 \pm 0.02$ & $0.23 \pm 0.02$ & 0.922 \\
\hline
\end{tabular}

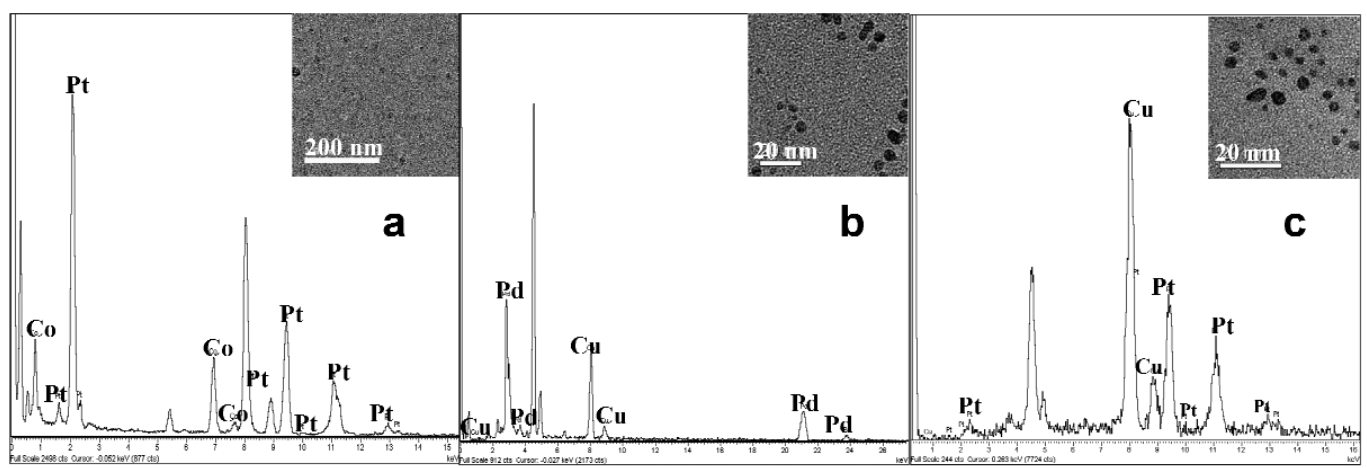

Fig. 3 Energy-dispersive spectra (EDS) of Pt@Co-, Pd@Cu-, and Pt@Cu-PSMNPs. Insets show TEM images of MNP samples corresponding to each EDS.

The TEM images obtained have been used to calculate the size-distribution histograms corresponding to different PSMNPs samples. The results obtained by fitting the histogram with a 3-parameter Gaussian curves (see eq. 7), are presented in Tables $1-4$.

$$
y=a \cdot \exp \left\{-0.5\left[\frac{\left(\mathrm{d}-\mathrm{d}_{m}\right)}{\sigma}\right]^{2}\right\}
$$

Here, $a$ is the height of Gaussian peak [which corresponds to $1 /\left(2 \pi \sigma^{2}\right)^{1 / 2}$ ], $d_{\mathrm{m}}$ is the position of the center of the peak (corresponding to the most frequent diameter), and $\sigma$ is the standard deviation.

Table 2 MNP sizes and parameters of histograms corresponding to different Pt@ $\mathrm{Cu}-\mathrm{MNP}$ samples.

\begin{tabular}{lccccc}
\hline $\begin{array}{l}\text { Sample } \\
\text { no. }\end{array}$ & $\begin{array}{c}\text { mmols Pt/g SPEEK in } \\
2^{\text {nd }} \text { loading solution }\end{array}$ & $a$ & $d_{\mathrm{m}}(\mathrm{nm})$ & $\sigma(\mathrm{nm})$ & $r^{2}$ \\
\hline A2 & 0.33 & $184 \pm 21$ & $3.38 \pm 0.11$ & $0.83 \pm 0.11$ & 0.941 \\
A3 & 0.46 & $53 \pm 3$ & $4.31 \pm 0.11$ & $1.44 \pm 0.11$ & 0.980 \\
A4 & 0.52 & $210 \pm 19$ & $3.50 \pm 0.08$ & $0.84 \pm 0.09$ & 0.961 \\
A5 & 0.65 & $234 \pm 12$ & $3.29 \pm 0.04$ & $0.65 \pm 0.04$ & 0.990 \\
A7 & 1.30 & $175 \pm 7$ & $4.03 \pm 0.04$ & $0.95 \pm 0.04$ & 0.990 \\
\hline
\end{tabular}


Table 3 MNP sizes and parameters of histograms corresponding to different Pd@CuMNP samples.

\begin{tabular}{lcrccr}
\hline $\begin{array}{l}\text { Sample } \\
\text { no. }\end{array}$ & $\begin{array}{c}\text { mmols Pd/g SPEEK in } \\
2^{\text {nd }} \text { loading solution }\end{array}$ & $a$ & $d_{\mathrm{m}}(\mathrm{nm})$ & $\sigma(\mathrm{nm})$ & $r^{2}$ \\
\hline B1 & 0.19 & $22 \pm 3$ & $2.66 \pm 0.10$ & $0.76 \pm 0.10$ & 0.857 \\
B3 & 0.46 & $240 \pm 4$ & $3.34 \pm 0.01$ & $0.79 \pm 0.01$ & 0.999 \\
B4 & 0.52 & $293 \pm 7$ & $4.52 \pm 0.03$ & $1.20 \pm 0.03$ & 0.995 \\
B5 & 0.65 & $129 \pm 4$ & $4.60 \pm 0.05$ & $1.37 \pm 0.05$ & 0.991 \\
B7 & 1.30 & $110 \pm 9$ & $4.90 \pm 0.15$ & $1.65 \pm 0.15$ & 0.934 \\
\hline
\end{tabular}

Table 4 MNP sizes and parameters of histograms corresponding to different Pd-MNP samples.

\begin{tabular}{lccccc}
\hline $\begin{array}{l}\text { Sample } \\
\text { no. }\end{array}$ & $\begin{array}{c}\text { mmols Pd/g SPEEK in } \\
2^{\text {nd }} \text { loading solution }\end{array}$ & $a$ & $d_{\mathrm{m}}(\mathrm{nm})$ & $\sigma(\mathrm{nm})$ & $r^{2}$ \\
\hline D1 & 0.19 & $43 \pm 4$ & $6.1 \pm 0.2$ & $2.0 \pm 0.2$ & 0.904 \\
D3 & 0.46 & $87 \pm 6$ & $7.2 \pm 0.15$ & $2.03 \pm 0.15$ & 0.947 \\
D4 & 0.52 & $147 \pm 3$ & $7.22 \pm 0.03$ & $1.14 \pm 0.03$ & 0.996 \\
D5 & 0.65 & $173 \pm 4$ & $8.08 \pm 0.05$ & $1.88 \pm 0.05$ & 0.992 \\
D7 & 1.30 & $27 \pm 2$ & $12.14 \pm 0.09$ & $1.42 \pm 0.09$ & 0.958 \\
\hline
\end{tabular}

The size of Pt@Cu-, Pd@Cu-, and Pd-PSMNPs, which have been synthesized by using different Pt or Pd loading concentrations (second metal loading-reduction cycle), and the parameters of corresponding histograms are shown in Tables $2-4$.

As it clearly follows from the results presented in Tables 2 and 3, the coating of $\mathrm{Cu}$ with $\mathrm{Pt}$ first decreases the size of MNPs (considering that Cu-MNP size is around $7 \mathrm{~nm}$ ) followed by a slight growth of MNP size (see sample A7 in Table 2), however, in the case of Pd@Cu (see Table 3) this effect is not observed. Two possible reasons can be distinguished to be responsible for this effect [15]. The first one may deal with the partial oxidation of $\mathrm{Cu}$-core-MNPs during the loading of $\mathrm{Cu}$-PSMNP-containing membrane with $\left[\mathrm{Pt}\left(\mathrm{NH}_{3}\right)_{4}\right]\left(\mathrm{NO}_{3}\right)_{2}$ solution. The $\mathrm{Cu}$ oxidation reaction in this case can be written as follows:

$$
\mathrm{Cu}^{0}(\mathrm{~s})+\left[\mathrm{Pt}\left(\mathrm{NH}_{3}\right)_{4}\right]^{2+} \rightarrow\left[\mathrm{Cu}\left(\mathrm{NH}_{3}\right)_{4}\right]^{2+}+\mathrm{Pt}^{0} @ \mathrm{Cu}^{0}(\mathrm{~s})
$$

The second possible reason can be associated with an increase of the interior pressure in bimetallic core-shell nanoparticles compared with that in the monometallic ones [15,24,25].

Synthesis of catalytically active core-shell MNPs is particularly important for two reasons: (i) the smaller size of core-shell PGM-containing MNPs results in the increase of their surface area, which is extremely important for their use in heterogeneous catalysis; and (ii) the cost of core-shell MNPs appears to be far lower than that of their monometallic analogs due to the use of cheap core metals (e.g., $\mathrm{Cu}, \mathrm{Co}$, etc.) and relatively low loading of PGM.

As is clearly seen from the results shown in Table 2, the size of Pt@Cu-MNPs does not change dramatically with variation of concentration of the Pt loading solution. Indeed, the change of coreshell MNP diameters in this case does not exceed $30 \%$. Comparison of the results presented in Table 2 with those shown in Table 3 allows for the conclusion that a far higher increase of MNP sizes is observed in the case of Pd@Cu-MNPs. The diameters of corresponding MNPs increase up to $84 \%$ with concentration of Pd loading solution. However, the maximum change of MNP sizes with concentration of the loading solution is observed in the case of monometallic Pd-PSMNPs (see Table 4). These results can also be explained by considering the above two factors: (i) partial compression of $\mathrm{Cu}$-core after the coating with PGM shell and (ii) partial oxidation of the core in the course of the second load- 
ing with PGM solution. For Pd, both the value of standard reduction potential, $E^{\circ}(\mathrm{Pd})$, and that of the surface tension are less than the respective values for Pt $[15,26]$. Therefore, both effects have to be less pronounced in the case of Pd@Cu- in comparison with Pt@Cu-MNPs. Unlike bimetallic core-shell MNPs, the size of monometallic Pd-MNPs gradually increases with concentration of the Pd loading solution.

Table 5 shows the compositions of Pt@Cu-MNP samples determined by using inductively coupled plasma-optical emission spectroscopy (ICP-OES) technique after the treatment of corresponding nanocomposite membranes with Aqua Regia. So, the $\mathrm{Cu}$ content in all PSMNP-SPEEK nanocomposite membranes remains practically constant while that of Pt increases with Pt concentration in the loading solution.

Table $5 \mathrm{Cu}$ and Pt composition of Pt@Cu-SPEEK membranes.

\begin{tabular}{lcr}
\hline Sample no. & mg Cu/g SPEEK & $\mathrm{mg} \mathrm{Pt} / \mathrm{g}$ SPEEK \\
\hline A2 & $51.2 \pm 0.2$ & $46.8 \pm 0.4$ \\
A3 & $57.7 \pm 0.2$ & $62.0 \pm 0.4$ \\
A4 & $54.4 \pm 0.3$ & $66.4 \pm 0.4$ \\
A5 & $52.2 \pm 0.2$ & $88.4 \pm 0.6$ \\
A7 & $51.5 \pm 0.2$ & $152.5 \pm 0.1$ \\
\hline
\end{tabular}

The results shown in Tables 2 and 5 allow for calculation of the structural parameters of Pt@ $\mathrm{Cu}-$ MNPs (the radius of $\mathrm{Cu}$-core, $r_{\mathrm{Cu}}$, and the thickness of the Pt-shell, $\left.1_{\mathrm{Pt}}\right)$ and the number of MNPs $\left(N_{\mathrm{np}}\right)$ in one gram of PSMNP-SPEEK nanocomposites, by using the following simple model:

$$
\begin{aligned}
& d_{\mathrm{Cu}}+21_{\mathrm{Pt}}=d_{\mathrm{m}} \\
& 4 / 3 \cdot \pi \cdot r_{\mathrm{Cu}}{ }^{3} \cdot N_{\mathrm{np}}=V_{\mathrm{Cu}} \\
& 4 / 3 \cdot \pi \cdot\left(r_{\mathrm{m}}{ }^{3}-r_{\mathrm{Cu}}{ }^{3}\right) \cdot N_{\mathrm{np}}=V_{\mathrm{Pt}}
\end{aligned}
$$

Here, $r_{\mathrm{m}}=d_{\mathrm{m}} / 2$ and $d_{\mathrm{m}}$ is the MNP diameter corresponding to the maximum on respective size-distribution histogram; $N_{\mathrm{np}}$ is the number of nanoparticles per one gram of membrane; and $V_{\mathrm{Cu}}$ and $V_{\mathrm{Pt}}$ are the volume of respective metal $(\mathrm{Cu}$-core and Pt-shell) per $1 \mathrm{~g}$ of membrane. The results of calculation of the structural paramers of Pt@Cu-PSMNP samples by using eqs. 9-11 are shown in Table 6. As seen, the diameter of $\mathrm{Cu}$-core in all core-shell PSMNP samples appears to be essentialy constant while the thickness of Pt layer increases with Pt loading.

Table 6 Structural parameters of Pt@Cu-MNPs calculated by using eqs. 9-11.

\begin{tabular}{lccc}
\hline Sample no. & $d_{\mathrm{Cu}}(\mathrm{nm})$ & $N_{\mathrm{np}} \cdot 10^{17} / \mathrm{g}$ membrane & $\ell_{\mathrm{Pt}}(\mathrm{nm})$ \\
\hline A2 & 3,1 & 5.0 & 0.17 \\
A3 & 3.8 & 2.9 & 0.25 \\
A4 & 3,1 & 5.3 & 0.22 \\
A5 & 2.7 & 7.1 & 0.27 \\
A7 & 3,1 & 5.1 & 0.45 \\
\hline
\end{tabular}

\section{Study of PSMNP stability}

As discussed in the introduction, the main drawback of MNPs is their instability due to the high tendency for aggregation. When using IMS technique, the polymer acts as esteric stabilizer, which serves as a barrier preventing coalescence and aggregation of PSMNPs. The mechanism of nanoparticle sta- 
bilization in this case can be attributed to two factors: (i) a high viscosity of the polymer, which prevents migration of MNPs inside polymeric matrix, and (ii) a decrease of the energy of inter-particle interaction [2,6]. The PSMNPs can be stored in two forms: (i) as nanocomposite-membranes, or (ii) as a PSMNP ink in case of the use of non-cross-linked polymers (e.g., SPEEK) due to their solubility in some solvents (e.g., DMF). It seems clear that the stability of PSMNPs in the first case is far higher than that in the second one due to the incomparably higher viscosity of the solid polymer in comparison with its solution. However, TEM analysis requires preparation of sufficiently diluted solution (or suspension) of PSMNPs to prevent the formation of polymeric film on the grid (object support), which makes it essentially impossible to obtain good MNP images applicable for the size-distribution measurements. For this reason, the PSMNP inks were used to study stability of nanoparticles in time. The results of this study are shown in Fig. 4, where size-distribution histograms, obtained right after dissolution of PSMNP-SPEEK nanocomposite membranes in DMF (freshly prepared) and after 7 months of ink storage under laboratory conditions for two Pt@Cu-PSMNP samples (A2 and A4, see Table 2) are shown.
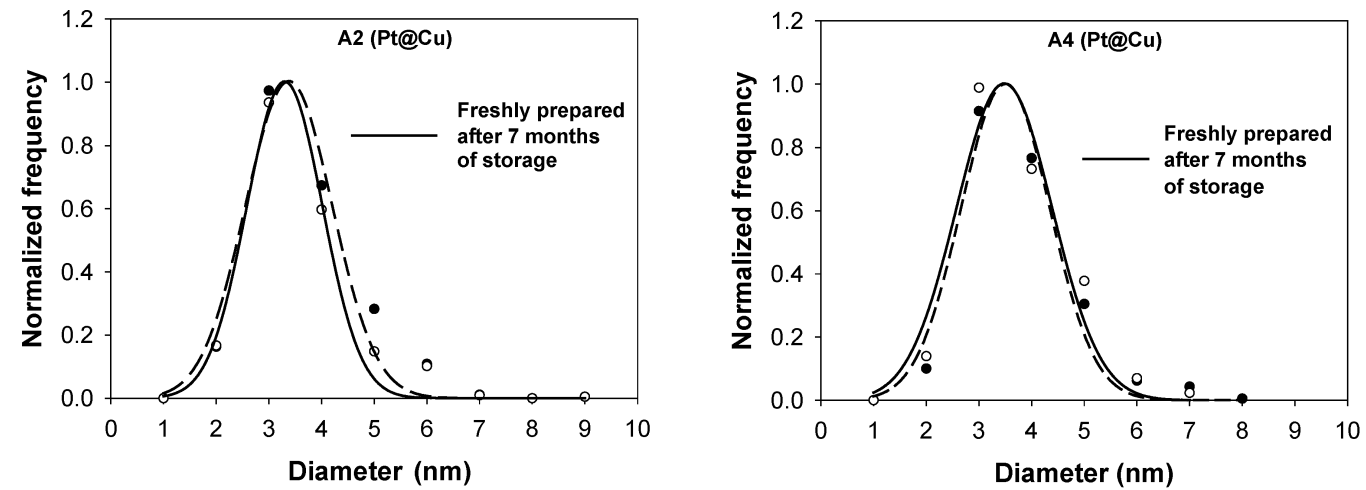

Fig. 4 Size-distribution histograms of freshly prepared Pt@Cu-PSMNP samples and after 7 months of storage $(\bigcirc)$ under ambient conditions.

The size-distribution histograms, presented in Fig. 4, were obtained for PSMNP inks (5 \% mass solution in DMF) by using periodical TEM analysis and the measurement of PSMNP sizes. As seen, the shape of histograms remains essentially unchanged even after 7 months of storage of PSMNP solutions (PSMNP inks). The results obtained testify to the extremely high stability of the inks, which confirms a very high stabilizing efficiency of SPEEK matrix toward MNPs synthesized by using IMS technique. Another conclusion, which follows from these results, concerns the recommendations for PSMNP storage conditions. Indeed, as it clearly follows from the results shown in Fig. 4, the PSMNP inks appear to be stable at least for half a year, however, the stability (or life-time) of nanoparticles inside solid SPEEK membranes has to be far higher. For this reason, PSMNPs have to be better stored in the form of solid membranes and dissolved before their application.

\section{Electrochemical characterization}

\section{PSMNP-based sensors}

The calibration curves illustrating the performance of GECE modified with Pt@Cu,Pd@Cu, Pt@Co-, Pd@Co-, Pt@Ni-, Pd@Ni-, and Pd@CoNi-PSMNP composite membranes in amperometric detection of $\mathrm{H}_{2} \mathrm{O}_{2}$ are shown in Figs. 5 and 6. The sensitivity of sensors toward analyte under study increases in the following order: 


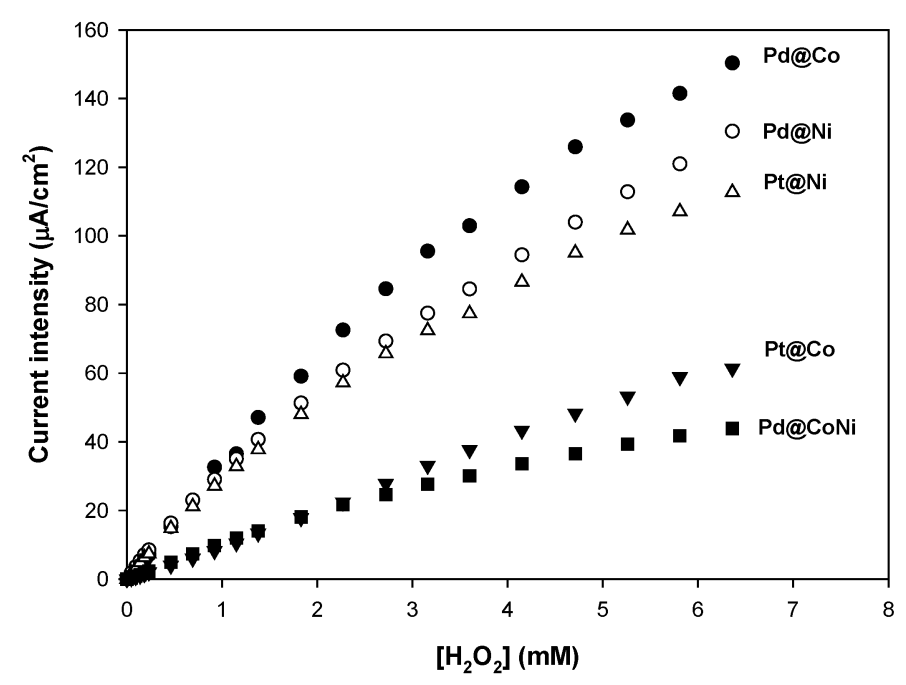

Fig. 5 Calibration curves of electrochemical detection of $\mathrm{H}_{2} \mathrm{O}_{2}$ concentration with GECEs modified with Pt@Co-, Pd@Co-, Pt@Ni-, Pd@Ni-, and Pd@CoNi-PSMNP composite membranes. Experimental conditions: potential: $-250 \mathrm{mV} ; 0.1 \mathrm{M}$ acetate buffer, $\mathrm{pH}=5.0$.

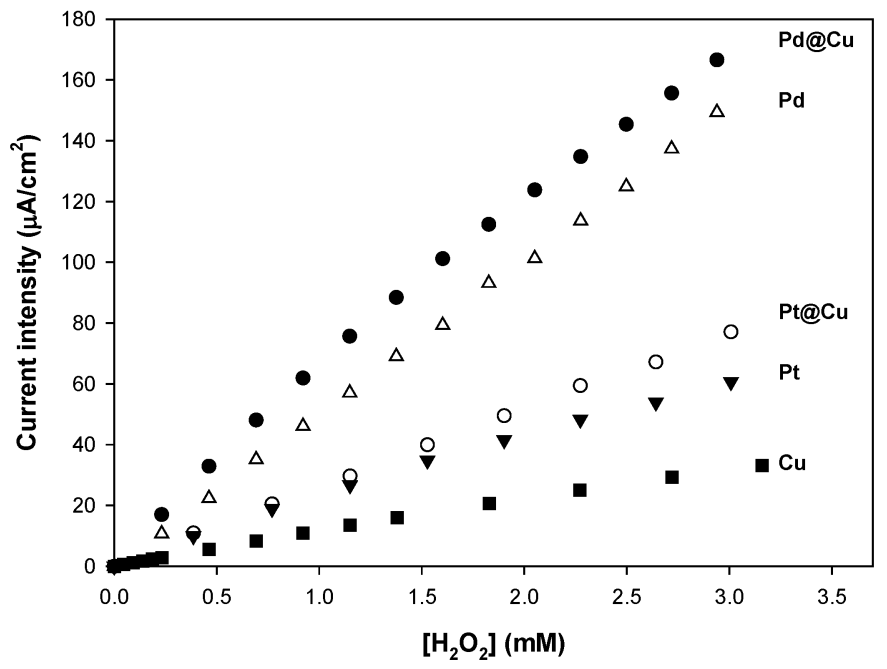

Fig. 6 Calibration curves of electrochemical detection of $\mathrm{H}_{2} \mathrm{O}_{2}$ concentration with $\mathrm{Pt} @ \mathrm{Cu}-, \mathrm{Pd} @ \mathrm{Cu}-, \mathrm{Pt}-, \mathrm{Pd}-$, and Cu-PSMNP composite membranes (with $0.194 \mathrm{mmol}$ of Pt or Pd per g SPEEK in all cases). Experimental conditions: potential: $-250 \mathrm{mV} ; 0.1 \mathrm{M}$ acetate buffer.

PSMNPs with ferromagnetic core:

Pd@Co >Pd@Ni > Pt@Ni > Pt@Co > Pt@Co-Ni (see Fig. 5)

PSMNPs with diamagnetic core:

$\mathrm{Pd} @ \mathrm{Cu}>\mathrm{Pd}>\mathrm{Pt} @ \mathrm{Cu}>\mathrm{Pt}>\mathrm{Cu}$ (see Fig. 6)

Note that the synthetic conditions of preparation of PSMNPs with ferromagnetic and diamagnetic cores differ from each other. However, they have been maintained constantly for each series of PSMNPs. The results presented in Figs. 5 and 6 demonstrate that the sensors based on the core-shell 
MNPs with Pd shell have the highest sensitivity. This can be the result of a higher catalytic activity of Pd in $\mathrm{H}_{2} \mathrm{O}_{2}$ decomposition than Pt-PSMNPs under the same experimental conditions.

Calibration curves obtained for sensors modified with monometallic Pd-, Pt-, Cu-, and bimetallic Pt@Cu- and Pd@Cu-PSMNPs indicate that bimetallic core-shell nanoparticles have a higher sensitivity than the monometallic ones (see Fig. 6). These results correlate well with the size of corresponding MNPs, as bimetallic nanoparticles are generally smaller than their monometallic analogs, and, therefore, have a higher specific surface area. The later parameter is known to be of particular importance in the heterogeneous catalytic systems.

\section{PSMNP-based glucose biosensors}

The glucose biosensor was prepared by using the layer-by-layer deposition technique. The GECE modified with SPEEK-Pt@Cu-PSMNPs membrane was sequentially treated with PEI and GOx solutions, which form intermittent monolayers of "sandwich-like" structure as is shown schematically in Fig. 7. The use of layer-by-layer deposition makes it possible to accumulate the amount of the enzyme inside the top part of the biosensor membrane, which provides the desired sensitivity.

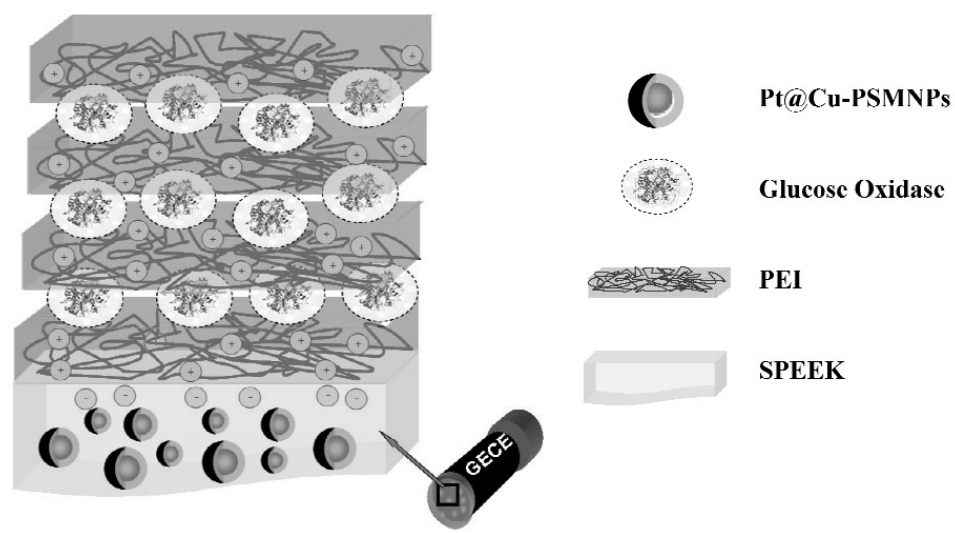

Fig. 7 Schematic diagram of supramolecular construction of glucose biosensor based on SPEEK-Pt@Cu-PSMNP nanocomposite.

On the other hand, as is clearly seen in Fig. 8, the sensitivity of biosensor at the same enzyme content (6 PEI-GOx layers) appears to strongly depend on the thickness of Pt-shell. This means that the thickness of the catalytically active shell of MNPs can be used as an additional parameter for enhancing the biosensor performance. This conclusion follows from a high sensitivity of Pt@ $\mathrm{Cu}$ toward hydrogen peroxide, which is one of the by-products of the enzymatic reaction proceeding in the course of glucose detection. From this viewpoint, MNPs immobilized in the bottom SPEEK membrane of the biosensor (see Fig. 7) fulfill in fact two functions: (1) they serve as the mediators, and (2) they enhance the electric conductivity of the polymer-metal nanocomposite membrane. Note that even better results (higher biosensor sensitivity) can be expected for biosensors prepared by using Pd@Cu-PSMNPs (see Fig. 6) and we follow in our study in this direction. 


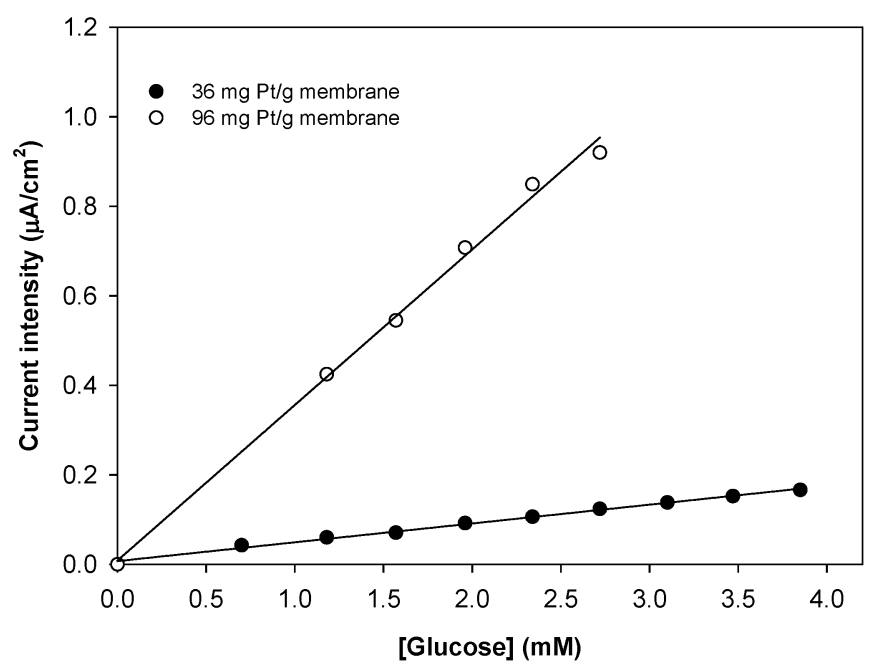

Fig. 8 Calibration curves of amperometric detection of glucose with GECE biosensors modified with SPEEK$\mathrm{Pt} @ \mathrm{Cu}-\mathrm{PSMNP}$ membranes and $6 \mathrm{PEI}-\mathrm{GOx}$ "sandwiches". Conditions: potential $=-250 \mathrm{mV} 0.1 \mathrm{M} \mathrm{KCl}$ in $0.1 \mathrm{M}$ acetate buffer at $\mathrm{pH}=7.0$.

\section{CONCLUSIONS}

Intermatrix synthesis technique allows for the synthesis of both mono- and bimetallic core-shell PSMNPs by using a simple and inexpensive procedure. The IMS technique is based on the use of functionalized polymeric membrane as a nanoreactor to both synthesize and characterize PSMNPs of desired structure and composition. The synthesis of core-shell PSMNPs includes two sequential metal loading-reduction cycles, the first of which is used for the formation of the core MNPs while the second one serves for their coating with the layer (shell) of the second metal. Under the same IMS conditions, the surface area of monometallic PSMNPs appears to be lower than that of their bimetallic coreshell analogs. The nanoparticles obtained by IMS have high storage stability both in the form of polymer-metal nanocomposite membranes and PSMNP inks that is the result of high stabilizing efficiency of SPEEK matrix. The amperometric sensors prepared by modification of GECE with the PSMNP inks demonstrate a high sensitivity toward hydrogen peroxide. The sensitivity of Pt@CuPSMNP-based glucose biosensor appears to depend on the thickness of Pt-shell at the same enzyme loading.

\section{ACKNOWLEDGMENTS}

This work was supported by the research grants INTAS Ref. No. 05-1000008-7834 and MAT200603745, 2006-2009 from the Ministry of Science and Technology of Spain, which is also acknowledged for financial support of Dmitri N. Muraviev within the Program Ramon y Cajal. Special thanks are given to Servei de Microscopia from Universitat Autònoma de Barcelona. Jorge Macanás thanks the support of Dept. d'Educació i Universitats de la Generalitat de Catalunya for a postdoctoral grant. 


\section{REFERENCES}

1. A. Bhakta, E. Ruckenstein. J. Chem. Phys. 103, 7120 (1995).

2. A. D. Pomogailo, A. S. Rozenberg, I. E. Uflyand. Metal Nanoparticles in Polymers, Khimia, Moskow (2000) (in Russian).

3. A. D. Pomogailo. Russ. Chem. Rev. 69, 53 (2000).

4. A. D. Pomogailo, G. I. Dzhardimalieva, A. S. Rozenberg, D. N. Muraviev. J. Nanoparticle Res. 5, 497 (2003).

5. A. D. Pomogailo. Usp. Khim. (Russ. Chem. Rev.) 66, 679 (1997).

6. D. N. Muraviev. Contribut. Sci. 3, 19 (2005).

7. D. N. Muraviev, J. Macanas, M. J. Esplandiu, M. Farre, M. Muñoz, S. Alegret. Phys. Status Solidi A 204, 1686 (2007).

8. J. Macanas, J. Parrondo, M. Muñoz, S. Alegret, F. Mijangos, D. N. Muraviev. Phys. Status Solidi A 204, 1699 (2007).

9. M. Antonietti, E. Wenz, L. Bronstein, M. Seregina. Adv. Mater. 7, 1000 (1995).

10. B. Corain, M. Kralik. J. Mol. Catal. A 159, 153 (2000).

11. M. Somasundrum, K. Kirtikara, M. Tanticharoen. Anal. Chim. Acta 319, 59 (1996).

12. J. Wang. Analyst 130, 421 (2005).

13. F. Caruso. Adv. Mater. 13, 11 (2001).

14. J. H. Fendler. Nanoparticles and Nanostructured Films, Wiley-VCH, Weinheim, (1998).

15. D. N. Muraviev, J. Macanas, J. Parrondo, M. Muñoz, A. Alonso, S. Alegret, M. Ortueta, F. Mijangos. React. Funct. Polym. 67, 1612 (2007).

16. D. N. Muraviev, J. Macanas, M. Farre, M. Muñoz, S. Alegret. Sens. Actuators, B 118, 408 (2006).

17. G. Kickelbick. Prog. Polym. Sci. 28, 83 (2003).

18. S. U. Son, Y. Jang, J. Park, H. B. Na, H. M. Park, H. J. Yun, J. Lee, T. Hyeon. J. Am. Chem. Soc. 126, 5026 (2004).

19. J. Macanas, M. Farre, M. Muñoz, S. Alegret, D. N. Muraviev. Phys. Status Solidi A 203, 1194 (2006).

20. F. Cespedes, E. Martinez-Fabregas, S. Alegret. Trends Anal. Chem. 15, 296 (1996).

21. C. A. Linkous, H. R. Anderson, R. W. Kopitzke, G. L. Nelson. Int. J. Hydrogen Energy 23, 525 (1998).

22. M. C. Rodriguez, G. A. Rivas. Electroanalysis 16, 1717 (2004).

23. B. Nowack, T. D. Bucheli. Environ. Pollut. 150, 5 (2007).

24. S. H. Ehrman. J. Colloid Interface Sci. 213, 258 (1999).

25. T. Chraska, A. H. King, C. C. Berndt. Mater. Sci. Eng. A 286, 169 (2000).

26. W. J. Plieth. J. Phys. Chem. 86, 3166 (1982). 